\title{
Glucagon-like peptide-1 receptor agonists inhibit hepatic stellate cell activation by blocking the p38 MAPK signaling pathway
}

\author{
L.K. Wu' ${ }^{1}$, Y.C. Liu', L.L. Shi ${ }^{1}$ and K.D. Lu ${ }^{2}$ \\ ${ }^{1}$ The Second Clinical College, Zhejiang TCM University, Gongshu District, \\ Hangzhou, China \\ ${ }^{2}$ The First Clinical College, Zhejiang TCM University, Shangcheng District, \\ Hangzhou, China \\ Corresponding author: K.D. Lu \\ E-mail: Itaohong@163.com
}

Genet. Mol. Res. 14 (4): 19087-19093 (2015)

Received August 10, 2015

Accepted October 7, 2015

Published December 29, 2015

DOI http://dx.doi.org/10.4238/2015.December.29.17

ABSTRACT. We investigated the effects of glucagon-like peptide-1 receptor (GLP-1R) agonists on p38 mitogen-activated protein kinase (MAPK) signaling during inhibition of hepatic stellate cell (HSC) activity. Human HSCs were cultured and morphologically identified. HSC samples were collected and randomly divided into three groups ( $N=20$ samples per group): a control group treated with high glucose (final concentration 25 $\mathrm{mM}$ ); a GLP-1R agonist group treated with liraglutide (final concentration $5 \mathrm{mM}$ ); and a p38-blocked group treated with the p38 MAPK inhibitor SB203580 (final concentration $14 \mu \mathrm{M}$ ). All cells were cultured for $120 \mathrm{~h}$ followed by detection of phosphorylated p38 MAPK (p-p38 MAPK) and $\alpha$-smooth muscle actin ( $\alpha-S M A$, a measure of HSC activation) by western blot. p-p38 MAPK and a-SMA expression levels were both significantly lower in HSCs in the GLP-1R agonist and p38-blocked groups compared with the control group (all $P<0.01$ ). GLP-1R agonists may inhibit the activation of HSCs by blocking the p38 MAPK signaling pathway.

Key words: Hepatic stellate cell; $\alpha$-smooth muscle actin; p38 mitogen-activated protein kinase signaling pathway;

Glucagon-like peptide-1 receptor agonist 


\section{INTRODUCTION}

Fatty liver disease specific to type 2 diabetes is called diabetic type 2-associated fatty liver (DFL), and it is a common complication of type 2 diabetes. Patients with diabetes have a high incidence of liver fibrosis caused by DFL and this has become a major cause of liver cirrhosis in China. The main clinical treatment for diabetes and DFL involves regulating blood sugar and lipids, but there is currently no satisfactory treatment for liver fibrosis caused by diabetes. GLP-1 is an incretin secreted from liver cells in the ileum and colon, which can stimulate pancreatic beta cells to secrete insulin at high blood glucose levels. GLP-1R agonists such as liraglutide and exenatide, which directly target GLP-1Rs, have recently received attention for the treatment of type 2 diabetes. Furthermore, interest in DPP-4 inhibitors such as sitagliptin and vildagliptin, which target GLP-1Rs indirectly, has also increased because of their convenience as oral formulations. These drugs can exert direct or indirect effects on pancreatic beta cells, stimulating them to secrete insulin through downstream signal transduction. They can also improve the function and inhibit apoptosis of pancreatic beta cells; therefore, these drugs are of interest for the treatment of type 2 diabetes.

Glucagon-like peptide-1 receptor (GLP-1R) agonists and dipeptidyl peptidase 4 (DPP-4) inhibitors are antidiabetic agents that directly or indirectly target the GLP-1R, and have recently been widely used in clinical settings. GLP-1Rs are expressed not only bypancreatic beta cells but also in other tissues such as heart, kidney, and lung. It was recently found that GLP-1 demonstrated antagonistic effects on fibrosis in multiple organs, including the liver. The key step in liver fibrosis involves hepatic stellate cell (HSC) activation leading to excessive deposition of extracellular matrix, suggesting that the antagonistic effect of GLP-1 on hepatic fibrosis may involve the signal transduction pathways that regulate HSC activation. Alpha-smooth muscle actin ( $\alpha-S M A)$ protein expression levels are a measure of HSC activation. Given the close relationship between the p38 mitogen-activated protein kinase (MAPK) signaling pathway and the activation of HSCs, we focused on the role of this pathway in the mechanism of GLP-1R agonist-mediated inhibition of HSC activation.

\section{MATERIAL AND METHODS}

\section{Cell lines and reagents}

The HSC line LI-90 was obtained from the Experimental Animal Center of Sun Yat-sen University (Guangzhou, China). L-Dulbecco's modified Eagle's medium (DMEM) was purchased from Gibco (USA). Fetal calf serum was purchased from Harry Bio-Engineering Co. Ltd. (Chengdu, China). The p38 MAPK inhibitor SB203580 was purchased from New England Biolabs (USA). Primary antibodies and the corresponding species labeled secondary antibodies for $\alpha$-SMA, phospho (p)-p38 MAPK and GAPDH were purchased from Boster Bio-Engineering Co. Ltd. (Wuhan, China). The enhanced chemiluminescence kit was purchased from KGI Bio-Technology Development Co. Ltd. (Nanjing, China). Liraglutide was purchased from Novo Nordisk (Denmark).

\section{Grouping}

HSC samples were collected and randomly divided into three groups ( $N=20$ for each group): a control group treated with high glucose (final concentration $25 \mathrm{mM}$ ); a GLP-1R agonist group treated with liraglutide (final concentration $5 \mathrm{mM}$ ); and a p38-blocked group, treated with the p38 MAPK blocking agent SB203580 (final concentration $14 \mu \mathrm{M}$ ). All cells were cultured for $120 \mathrm{~h}$ and then used in subsequent experiments. 


\section{Experimental methods}

\section{HSC culture}

After recovery, HSCs were seeded in 2.5-mL plastic culture flasks. HSCs were routinely cultured in a $5 \% \mathrm{CO}_{2}$, humidified incubator at $37^{\circ} \mathrm{C}$. The culture medium was L-DMEM containing $10 \%(\mathrm{v} / \mathrm{v})$ fetal bovine serum (Gibco, USA) and the media were changed every 3 days.

\section{Analysis of HSC morphology}

HSCs were examined under an inverted microscope, and appeared flat with large cell bodies and well-developed stress fibers. They also lacked cytoplasmic lipid droplets.

\section{Extraction and quantification of sample protein}

HSC lysates were collected using a buffer containing $8 \mathrm{M}$ urea, 4\% CHAPS, 1\% tributyl phosphate (TBP), $0.2 \%$ Biolyte, and $0.001 \%$ bromophenol blue. After an ice bath with intermittent ultrasound and $15,000 \mathrm{~g}$ high-speed centrifugation at $4^{\circ} \mathrm{C}$, cell supernatants were obtained, and the protein concentration was estimated using a Bradford assay.

\section{Detection of $\alpha-S M A$ or p-p38 MAPK expression in human HSCs by western blot}

Proteins were separated using 10\% SDS-PAGE with Tris-glycine running buffer. After electrophoresis, the protein bands were transferred to $350 \mathrm{~mA}$ for $1 \mathrm{~h}$ onto a PVDF membrane. The membrane was then rinsed with TBS for $10 \mathrm{~min}$. The PVDF membrane was incubated for $1 \mathrm{~h}$ at room temperature in blocking solution (5\% skim milk in 1X TBS-T). The membranes were rinsed in $1 X$ TBS-T three times for 5 min. The PVDF membrane was incubated at $4^{\circ} \mathrm{C}$ overnight in the a-SMA or p-p38 MAPK primary antibody diluted in blocking solution (1:100 or 1:1000, respectively). The next day, the membrane was rinsed three times for 5 min in $1 X$ TBS-T. The PVDF membrane was incubated at $37^{\circ} \mathrm{C}$ for $1 \mathrm{~h}$ in horseradish peroxidase-conjugated secondary antibodies diluted in blocking solution (1:3000 for $\alpha$-SMA and 1:2000 for p-p38 MAPK) while shaking. The membrane was rinsed with $1 \mathrm{X}$ TBS-T three times for $5 \mathrm{~min}$ and washed once with TBS. The PVDF membrane was visualized using an enhanced chemiluminescence kit. The film was exposed, developed, and fixed, and analyzed by a transilluminator and its software (BD Biosciences, USA). GAPDH was used as an internal reference for semi-quantitative analysis.

\section{Statistical analysis}

Statistical analysis was conducted using the SPSS 17.0 software (Chicago, IL, USA). Statistical data were normally distributed. The Student $t$-test for two independent samples was used for statistical analysis

\section{RESULTS}

\section{Expression of p-p38 MAPK}

p-p38 MAPK protein expression levels in each group were detected by western blot using GAPDH as an internal reference (Figure 1). 


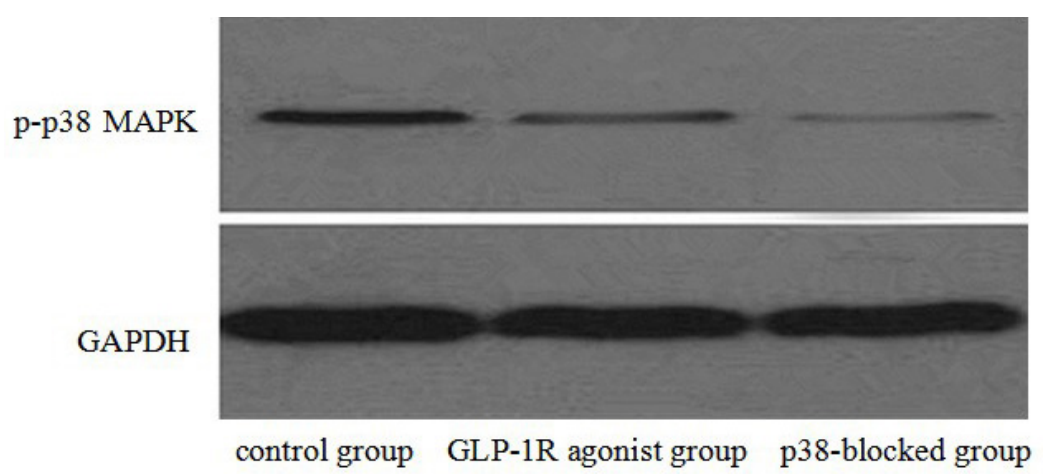

Figure 1. Expression levels of p-p38 MAPK protein in each group (control, GLP-1R agonist, and p38-blocked) detected by western blot. GAPDH is shown as an internal control. Densitometric analysis is shown in Table 1.

\section{Expression of $\alpha-S M A$ in each treatment group}

a-SMA protein expression levels are a measure of HSC activation and their levels in each group were detected by western blot with GAPDH as an internal reference (Figure 2).

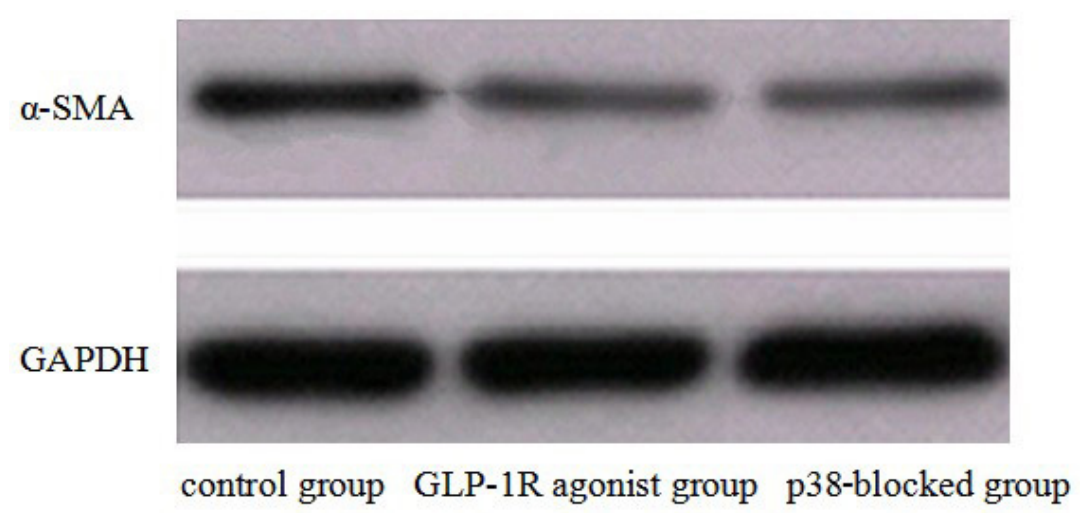

Figure 2. Expression levels of a-SMA protein in each group (control, GLP-1R agonist, and p38-blocked) detected by western blot. GAPDH is shown as an internal control. Densitometric analysis is shown in Table 1.

\section{Statistical analysis of experimental data}

p-p38 MAPK protein expression levels were significantly lower in the GLP-1R agonist and p38-blocked groups compared with the control group (both $P<0.01$; Table 1). Similarly, $\alpha$-SMA expression levels were also significantly lower in the GLP-1R agonist and p38-blocked groups compared with the control group (both $P<0.01$; Table 1). These results demonstrate that $p$-p38 MAPK and $\alpha$-SMA expression levels were both significantly reduced by treatment with the GLP$1 R$ agonist liraglutide, suggesting that GLP-1R agonists can inhibit p38 MAPK signaling and HSC activation. $\alpha$-SMA expression levels were also reduced by the p38 blocker SB203580, suggesting that HSC activation can be inhibited by blocking the p38 MAPK pathway. Overall, these results indicate that GLP-1R agonists may inhibit the activation of HSCs by blocking the p38 MAPK pathway. 
Table 1. Densitometric analysis of $\alpha-S M A$ and p-p38 MAPK expression by using western blot (means \pm SD).

\begin{tabular}{lcc}
\hline Group & p-p38 MAPK/GAPDH (\%) & $\alpha$-SMA/GAPDH (\%) \\
\hline Control & $22.79 \pm 3.80$ & $34.61 \pm 4.07$ \\
GLP-1R agonist & $10.13 \pm 4.955^{\star}$ & $23.90 \pm 6.02^{\star}$ \\
p38-blocked & $8.66 \pm 4.15^{\Delta}$ & $26.48 \pm 4.41^{\Delta}$ \\
\hline
\end{tabular}

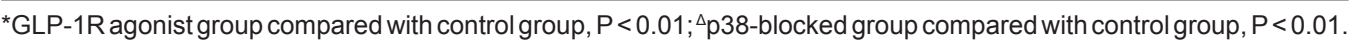

\section{DISCUSSION}

In the present study, we found p-p38 MAPK and a-SMA expression levels were both significantly lower in HSCs in the GLP-1R agonist and p38-blocked groups compared with the control group (all $P<0.01$ ). GLP-1R agonists may inhibit the activation of HSCs by blocking the p38 MAPK signaling pathway.

GLP-1Rs are not confined to the pancreas and have recently been shown to be widely expressed throughout the body. GLP-1Rs have been detected in the heart, liver, and thyroid, where they may play many important physiological roles (Gupta et al., 2010; Gier et al., 2012; Monji et al., 2013; Lockie, 2013). In addition to regulating insulin secretion in relation to blood sugar levels, GLP1 has also been shown to play protective and anti-fibrotic roles in the cardiovascular system. Park et al. (2007) treated 8-week-old male db/db mice with the long-acting GLP-1R agonist liraglutide for 8 weeks and found that the expression of transforming growth factor- $\beta 1$ and type IV collagen were significantly decreased. Hillman et al. (2012) recently found that GLP-1 levels were significantly reduced in cystic fibrosis patients $(P<0.01)$ compared with healthy individuals. In an electron microscope study, Tang et al. (2008) observed that the endothelial basement membrane thickness of pulmonary capillaries was significantly decreased following treatment with GLP-1 for 8 weeks in the Otsuka-Long-Evans-Tokushima fatty rat model $(P<0.01)$. The anti-fibrotic effects of GLP1 have thus been confirmed. We speculate that the mechanisms responsible for this anti-fibrotic effect are complex and may involve multiple pathways in different organs. Excessive deposition of extracellular matrix as a result of HSC activation is key in the pathology of liver fibrosis (Friedman, 2008a), which is regulated by the balance between HSC activation and apoptosis. Regulation of HSC activation can thus regulate the progress of liver fibrosis, and inhibition of HSC activation is important for halting, or even reversing, liver fibrosis (Baba et al., 2004; Russo et al., 2006; Roderfeld et al., 2006; De Minicis et al., 2007; Friedman, 2008b).

The mechanisms regulating HSC activation are extremely complex. Cell factors and activation products generated from liver cells, Kupffer cells, endothelial cells, and HSCs themselves, as well as environmental stimulation by drugs, can regulate the biological behavior of HSCs through various signaling pathways (Lin and Wang, 2006). The p38 MAPK signaling pathway was recently shown to play an important role in HSC stress, activation, proliferation, apoptosis, phenotypic transdifferentiation, and other physiological and pathological processes (Aouadi et al., 2006; Brown and Sacks, 2008; Kyriakis and Avruch, 2012). External signals can regulate HSC activation through the p38 MAPK signaling pathway and thus regulate hepatic fibrosis. This pathway includes signal transduction cascades that are initiated by extracellular signals and eventually lead to important intracellular biological effects. p-p38 MAPK is formed by the dual phosphorylation of specific threonine and tyrosine residues (Kim and Choi, 2010), after which it is rapidly transferred to the nucleus where it acts on the corresponding target to promote HSC activation (Tahashi et al., 2002). 
We previously demonstrated that GLP-1Rs were expressed in human HSCs (Wu et al., 2013), and it is known that GLP-1R stimulation can have an anti-fibrotic effect. We investigated the involvement of the p38 MAPK signaling pathway in fibrosis and demonstrated that the GLP1R agonist liraglutide could inhibit HSC activation by inhibiting p38-MAPK phosphorylation and consequently the p38 MAPK pathway. These results suggest that both GLP-1R agonists and DPP-4 inhibitors, which act indirectly on GLP-1Rs, could inhibit HSC activation via the p38-MAPK pathway and thus represent potential treatments for liver fibrosis. Further studies are needed to elucidate the mechanisms involved.

\section{Conflicts of interest}

The authors declare no conflict of interest.

\section{ACKNOWLEDGMENTS}

Research supported by the Zhejiang Provincial Health Science and Technology Program (\#2013KYA144).

\section{REFERENCES}

Aouadi M, Binetruy B, Caron L, Le Marchand-Brustel Y, et al. (2006). Role of MAPKs in development and differentiation: lessons from knockout mice. Biochimie 88: 1091-1098.

Baba S, Fujii H, Hirose T, Yasuchika K, et al. (2004). Commitment of bone marrow cells to hepatic stellate cells in mouse. J. Hepatol. 40: 255-260.

Brown MD and Sacks DB (2008). Compartmentalised MAPK pathways. Handb. Exp. Pharmacol. 2008: 205-235.

De Minicis S, Seki E, Uchinami H, Kluwe J, et al. (2007). Gene expression profiles during hepatic stellate cell activation in culture and in vivo. Gastroenterology 132: 1937-1946.

Friedman SL (2008a). Mechanisms of hepatic fibrogenesis. Gastroenterology 134: 1655-1669.

Friedman SL (2008b). Hepatic stellate cells: protean, multifunctional, and enigmatic cells of the liver. Physiol. Rev. 88: 125-172.

Gier B, Butler PC, Lai CK, Kirakossian D, et al. (2012). Glucagon like peptide-1 receptor expression in the human thyroid gland. J. Clin. Endocrinol. Metab. 97: 121-131.

Gupta NA, Mells J, Dunham RM, Grakoui A, et al. (2010). Glucagon-like peptide-1 receptor is present on human hepatocytes and has a direct role in decreasing hepatic steatosis in vitro by modulating elements of the insulin signaling pathway. Hepatology 51: 1584-1592.

Hillman M, Eriksson L, Mared L, Helgesson K, et al. (2012). Reduced levels of active GLP-1 in patients with cystic fibrosis with and without diabetes mellitus. J. Cyst. Fibros. 11: 144-149.

Kim EK and Choi EJ (2010). Pathological roles of MAPK signaling pathways in human diseases. Biochim. Biophys. Acta 1802: 396-405.

Kyriakis JM and Avruch J (2012). Mammalian MAPK signal transduction pathways activated by stress and inflammation:a 10year update. Physiol. Rev. 92: 689-737.

Lin XP and Wang XZ (2006). Liver fibrosis related factors and its function. Shijie Huaren Xiaohua Zazhi 14: 1037-1043.

Lockie SH (2013). The GLP-1 receptor in the brain:role in neuroendocrine control of energy metabolism and treatment target for obesity. J. Neuroendocrinol. 25: 597-604.

Monji A, Mitsui T, Bando YK, Aoyama M, et al. (2013). Glucagon-like peptide-1 receptor activation reverses cardiac remodeling via normalizing cardiac steatosis and oxidative stress in type 2 diabetes. Am. J. Physiol. Heart Circ. Physiol. 305: H295-304.

Park CW, Kim HW, Ko SH, Lim JH, et al. (2007). Long-term treatment of glucagon-like peptide-1 analog exendin-4 ameliorates diabetic nephropathy through improving metabolic anomalies in db/db mice. J. Am. Soc. Nephrol. 18: 1227-1238.

Roderfeld M, Weiskirchen R, Wagner S, Berres ML, et al. (2006). Inhibition of hepatic fibrogenesis by matrix metalloproteinase-9 mutants in mice. FASEB J. 20: 444-454.

Russo FP, Alison MR, Bigger BW, Amofah E, et al. (2006). The bone marrow functionally contributes to liver fibrosis. Gastroenterology 130: 1807-1821. 
Tahashi Y, Matsuzaki K, Date M, Yoshida K, et al. (2002). Differential regulation of TGF-beta signal in hepatic stellate cells between acute and chronicrat liver injury. Hepatology 35: 49-61.

Tang XY, Wang Y, Guo XH, Wang SH, et al. (2008). [Effects of glucagon like peptide-1 treatment on the alveolar capillary basal lamina in Otsuka Long-Evans Tokushima Fatty rats]. Beijing Da Xue Xue Bao 40: 178-180.

Wu LK, Li YM, Liu YC, Wang L, et al. (2013). The expression of glucagon-like peptide receptor in human hepatic stellate cell. Guoji Liuxingbingxue Chuanranbingxue Zazhi 40: 236-242. 